\title{
ДИСКУССИИ
}

\section{Финансовая составляющая отношений франчайзинга: проблема расчета ставки роялти}

\author{
Котляров И.Д. ${ }^{6}$
}

В предлагаемой статье разработана новая методика расчета ставки роялти с
учетом снижения риска деятельности франчайзи благодаря использованию интеллектуальной собственности франчайзера.

$J E L: G 34$

Ключевые слова: роялти, франчайзинг, франчайзер, франчайзи, риск

\section{Введение}

В настоящее время существуют различные методики определения величины роялти (регулярных платежей за право использования чужой интеллектуальной собственности в коммерческих целях), однако наибольшее распространение в бизнес-практике получила следующая формула:

$$
R=\frac{r}{100 \%} V
$$

где

$R$ - величина роялти, которые должен выплатить пользователь интеллектуальной собственности (ИС) ее владельцу, ден. ед.;

$r$ - ставка роялти, \%;

$V$ - объем продаж, полученный благодаря использованию ИС, ден. ед.

Для расчета ставки роялти применяется следующая модель:

$$
r=\frac{k P_{\text {sup }}}{P_{u s}} \cdot 100 \%=\frac{k\left(P_{u s}-P_{\text {ind }}\right)}{P_{u s}} \cdot 100 \%=\frac{k P_{\text {sup }}}{P_{\text {ind }}+P_{\text {sup }}},
$$

где

$k$ - доля владельца интеллектуальной собственности в сверхприбыли пользователя ИС;

$P_{\text {sup }}$ - сверхприбыль пользователя ИС, ден. ед.;

$P_{\text {ind }}$ - обычная прибыль независимого предпринимателя, занимающегося тем же видом бизнеса, что и пользователь ИС, ден. ед.;

$P_{u s}$ - общая прибыль пользователя ИС, ден. ед.

Определение величины $k$ происходит как на основе договоренностей между владельцем и пользователем ИС, так и с учетом сложившихся в соответствующей отрасли традиций. В случае лицензирования эксперты говорят о некотором усредненном значении $k$, равном 0,25.

Отметим, что формулы (1-2) применяются для расчета платы за право использования чужой ИС при всех формах торговли ИС. Однако, на наш взгляд, это не совсем корректно, поскольку при разных формах торговли ИС получаемая пользователем выгода от ИС обуславливается разными факторами, и эти факторы необходимо учитывать при определении величины ставки роялти.

\footnotetext{
${ }^{6}$ Доцент кафедры экономики фирмы Санкт-Петербургского филиала Национального исследовательского университета «Высшей школы экономики»
} 
В данной статье будет сделана попытка разработать механизм расчета платы за те преимущества, которые приобретает покупатель франшизы - франчайзи.

\section{Франчайзинг и лицензирование: анализ отличий}

Модель расчета роялти (1-2) традиционно используется при лицензировании и была механически перенесена на случай франчайзинга. Напомним определения этих экономических явлений.

Лицензия - разрешение на использование запатентованных изобретений, промышленных образцов, товарных знаков, знаков обслуживания, ноу-хау, авторских прав, предоставляемое правообладателем (лицензиаром) третьему лицу (лицензиату) на определенный срок, на определенной территории и на платной основе. Лицензирование собственно предоставление лицензии.

Франчайзинг - передача собственником (франчайзером) неисключительного права на использование своих торговой марки, товарного знака, знака обслуживания и фирменного наименования и связанных с ними технологий и ноу-хау другой компании (на определенный срок, на определенных условиях и на определенной территории), которая будет вести свою коммерческую деятельность только под переданной ей торговой маркой и в строгом соответствии с переданными ей технологиями. Набор объектов ИС, передаваемых при франчайзинге, называется франшизой, а компания, получающая право на их использование, - франчайзи.

Разница между франчайзингом и лицензированием состоит в том, что лицензиат, как правило, обладает собственной торговой маркой и своим ассортиментом товаров и услуг. После получения права на изготовление продукта по чужой технологии или на использование чужой торговой марки он от своих продуктов и торговых марок не отказывается, а лишь встраивает новый продукт в свой товарный ассортимент, а новую марку - в свой портфель брендов. После истечения срока действия лицензионного договора лицензиат остается со своим исходным портфелем продуктов и торговых марок и может продолжать самостоятельную коммерческую деятельность. Франчайзи же работает только по технологиям франчайзера и под его торговой маркой; своими товарами, торговой маркой и технологиями он не обладает, и создавать и развивать их в течение срока действия франчайзингового договора права не имеет. По окончании франчайзингового договора франчайзи не только теряет свой бизнес, но и в течение оговоренного периода не может работать в той же отрасли, с которым была связана использовавшаяся им франшиза.

Сверхприбыль лицензиата складывается из более высокой цены лицензионной продукции (обусловленной ее высоким качеством, известностью торговой марки, под которой она выпускается, и т.д.), а также из ее более низкой себестоимости (за счет получения от лицензиара более совершенных производственных технологий).

Аналогичный подход, как было сказано выше, применяют и к расчету ставки роялти при франчайзинге (Blair, Lafontaine, 2005; Алгазина, 2005, с. 12-14; Королева, 2006, с. 267268; Стажкова, 2007), т.е. предполагается, что роялти и в этом случае также представляет собой всего лишь изъятие части сверхприбыли франчайзи, обусловленной использованием ИС, предоставленной франчайзером. Однако, на наш взгляд, это принципиально неверно.

Дело в том, что при всем внешнем сходстве лицензирования и франчайзинга (в обоих случаях речь идет об использовании чужой ИС на возмездной основе) между этими явлениями существует важное различие: при лицензировании лицензиат лишь добавляет новые продукты в собственный товарный портфель, тогда как при франчайзинге речь идет о создании нового предприятия, которое будет работать исключительно под торговой маркой франчайзера. Таким образом, если в случае лицензирования речь действительно идет лишь о дополнительной прибыли лицензиата, то при франчайзинге франчайзи получает от франчайзера саму возможность ведения самостоятельного эффективного бизнеса. Интересно отметить, что именно этот аспект в теоретических работах, посвященных франчайзингу, 
рассматривается как ключевое преимущество франчайзинга, однако до сих пор, насколько нам известно, не делалось попыток его математической формализации для разработки корректного алгоритма расчета ставки роялти.

Как нам представляется, возможность ведения собственного эффективного бизнеса включает в себя две составляющие:

- Возможность получать больший доход по сравнению с нефранчайзинговыми предприятиями, предлагающими аналогичный товар (за счет более высокой цены, обусловленной наличием у франчайзи известной торговой марки; этот аспект роднит франчайзинг с лицензированием).

- Большая стабильность бизнеса и меньший риск неуспеха (благодаря использованию известной торговой марки и подтвердивших свою эффективность на практике коммерческих и производственных технологий, предоставленных франчайзером). Этот аспект франчайзинга достаточно подробно проанализирован в нашей статье (Котляров, 2007, с. 8487).

Это означает, что франчайзи должен не только делиться своим сверхдоходом с франчайзером, но и выплачивать ему вознаграждение за снижение риска своей деятельности.

Оговоримся, что в дальнейших рассуждениях для простоты будет рассматриваться механизм финансовых отношений между франчайзером и франчайзи, не предполагающий выплаты первоначального взноса. Однако, как будет показано ниже, полученная модель может быть легко доработана таким образом, чтобы она включала возможность взимания первоначального взноса.

\section{Уточненная модель расчета ставки роялти}

Необходимость учета в модели фактора риска делает целесообразным рассмотрение не ожидаемого дохода франчайзи $P_{f r}$, а его вероятностного дохода $V_{f r}$ :

$$
V_{f r}=W_{f r} P_{f r}
$$

где

$W_{f r}$ - вероятность получения дохода (на практике может определяться как средняя частота выживания франчайзи в течение всего срока действия договора франчайзинга).

Аналогично вводится вероятностный доход независимого предпринимателя $V_{\text {ind }}$ :

$V_{\text {ind }}=W_{\text {ind }} P_{\text {ind }}$,

где

$W_{\text {ind }}$ - вероятность получения независимым предпринимателем дохода $P_{\text {ind }}$. Обуславливается степенью выживания новых компаний в данном регионе.

Очевидно, что

$$
W_{f r} P_{f r}=\left(W_{\text {ind }}+W_{\text {sup }}\right)\left(P_{\text {ind }}+P_{\text {sup }}\right)
$$

где

$W_{\text {sup }}$ - дополнительная вероятность получения дохода (или, если выразиться несколько ненаучно, прирост безрисковости деятельности франчайзи по сравнению с независимым предпринимателем за счет получения такого инструмента привлечения потребителей, как известная торговая марка, а также эффективных коммерческих технологий франчайзера);

$P_{\text {sup }}$ - дополнительный доход, получаемый франчайзи от ведения деятельности под торговой марки франчайзера и с использованием его технологий (по сравнению с доходом независимого предпринимателя).

Раскрывая скобки в выражении (3), получаем

$$
W_{f r} P_{f r}=W_{\text {ind }} P_{\text {ind }}+W_{\text {sup }} P_{\text {ind }}+W_{\text {ind }} P_{\text {sup }}+W_{\text {sup }} P_{\text {sup }}
$$

Это означает, что ставка роялти должна рассчитываться по формуле 
(5)

$$
r=\frac{A W_{\text {ind }} P_{\text {ind }}+B W_{\text {sup }} P_{i n d}+C W_{\text {ind }} P_{\text {sup }}+D W_{\text {sup }} P_{\text {sup }}}{W_{\text {ind }} P_{i n d}+W_{\text {sup }} P_{i n d}+W_{\text {ind }} P_{\text {sup }}+W_{\text {sup }} P_{\text {sup }}} \cdot 100 \%
$$

где $A, D, C, D$ - доля франчайзера в соответствующем компоненте дохода франчайзи (в общем случае эти доли не равны). Аналогично можно ввести доли франчайзи в тех же компонентах своего дохода $a, b, c, d$, причем

$$
0 \leq A \leq 1, \quad 0 \leq a \leq 1, \quad A+a=1 .
$$

Аналогичные условия выполняются для остальных пар $B-b, C-c, D-d$.

Формула (4) показывает, что, в отличие от дохода лицензиата, доход франчайзи разлагается на четыре составляющих. Таким образом, для расчета ставки роялти необходимо разработать методы нахождения величин $A, D, C, D$ (будем называть их долевыми множителями). Проанализируем формулу (4) подробнее.

Очевидно, что в ее правой части можно выделить «смешанные» и «независимые» слагаемые. Независимыми слагаемыми будем называть те, нижние индексы у обеих частей которых совпадают (т.е. соответствующий компонент дохода франчайзи обеспечивается за счет усилий только одной стороны сделки франчайзинга - либо самого франчайзи, либо франчайзера), а смешанными - те, у которых нижние индексы различаются.

На взгляд автора, распределяться между участниками франчайзинговой сделки должны только те элементы дохода франчайзи, которые являются смешанными (иными словами, которые обеспечиваются совместными усилиями франчайзи и франчайзера), тогда как независимые компоненты должны целиком отходить той стороне франчайзинговой сделки, чьими усилиями они достигнуты. Это означает, что значение $A$ должно быть равно 0 (очевидно, что франчайзер не может претендовать на долю в доходе, который франчайзи получил бы в любом случае и без переданной ему ИС), а $D$ должно принимать значение 1 (поскольку эта доля дохода франчайзи целиком обуславливается наличием у него ИС, предоставленной франчайзером).

Отметим, что помимо метода расчета величины роялти как доли от оборота франчайзи существует и альтернативная методика, согласно которой роялти представляет собой фиксированную величину, не зависящую от объема продаж франчайзи. На наш взгляд, минимальный экономически оправданный размер этой фиксированной величины роялти $R_{f i x}$ может рассчитываться как:

$$
R_{\text {fix }}=W_{\text {sup }} P_{\text {sup }} \text {. }
$$

Если роялти меньше этой величины, то франчайзер не только не изымает часть сверхдохода франчайзи, но и недополучает свой собственный доход.

Что касается величин $B$ и $C$, то для их определения наиболее естественным представляется использовать следующую модель:

$$
B=\frac{W_{\text {sup }}}{W_{\text {sup }}+W_{\text {ind }}} \quad C=\frac{P_{\text {sup }}}{P_{\text {sup }}+P_{\text {ind }}} .
$$

Такой подход наиболее прост и прозрачен - величина долевого множителя пропорциональна вкладу франчайзера в общий доход и в общую вероятность выживания франчайзи соответственно. Однако следует помнить, что при его использовании чистый доход франчайзи может быть меньше, чем чистый доход независимого предпринимателя такая ситуация будет иметь место в том случае, если:

$$
W_{\text {ind }} P_{\text {ind }}+(1-B) W_{\text {sup }} P_{\text {ind }}+(1-C) W_{\text {ind }} P_{\text {sup }}<P_{\text {ind }}\left(W_{\text {ind }}+W_{\text {sup }}\right)
$$

или, что равнозначно, если:

$$
B W_{\text {sup }} P_{\text {ind }}>(1-C) W_{\text {ind }} P_{\text {sup }} \text {. }
$$

Однако будем исходить из предположения, что франчайзи, будучи заинтересованным в минимизации риска своей деятельности, согласен именно на такую модель распределения 
дохода, даже если это чревато для него потерями по сравнению с тем доходом, который он мог бы иметь, будучи независимым предпринимателем.

Таким образом, примем эту методику в качестве базовой.

Рассмотрим частные случаи методики (7).

1. $B=C=1$. Это означает, что:

$$
W_{\text {ind }}=0, P_{\text {ind }}=0
$$

или, в более мягкой и лучше соответствующей реальности форме:

$$
W_{\text {sup }}>W_{\text {ind }}, P_{\text {sup }}>>P_{\text {ind }} \text {. }
$$

На практике вряд ли целесообразно ожидать выполнения условия (8) даже в смягченной форме, поскольку это означает, что независимая предпринимательская деятельность на данном рынке невозможна. Если же условие (8) не выполняется, а франчайзер, тем не менее, установил именно такие значения этих долевых множителей, то это означает, что франчайзер, желая максимизировать свою прибыль, изымает у франчайзи весь дополнительный доход, получаемый им от использования ИС франчайзера. Такая модель может применяться в случае известной и престижной торговой марки франчайзера, гарантирующей успех на рынке, для которого характерны крайне низкая выживаемость и не менее низкий доход независимых предпринимателей. Хотя этот вариант задания значений множителей неудобен для франчайзи, тем не менее, он все равно выгоден для него поскольку фактически фирма франчайзи все равно выживет с вероятностью $W_{f r}$, а не $W_{\text {ind }}$, что минимизирует риски франчайзи. Чистый доход франчайзи $P_{f r}^{\text {net }}$ в этом случае равен:

$$
P_{f r}^{n e t}=P_{\text {ind }} \frac{W_{\text {ind }}}{W_{\text {ind }}+W_{\text {sup }}} .
$$

Следует указать, что именно на этом примере хорошо заметна разница между лицензированием и франчайзингом: лицензиат, как уже было сказано выше, заинтересован не в минимизации риска, а в росте дохода, и изъятие всего дополнительного дохода от использования лицензии для него неприемлемо, тогда как франчайзи, будучи в первую очередь заинтересованным в выживании своего бизнеса, на такое изъятие вполне может согласиться, и при этом все равно получит выгоду за счет снижения риска.

2. Множители $B$ и $C$ задаются следующим образом: один из них принимается равным 1, а второй рассчитывается по формуле (7). В этом случае франчайзер полностью изымает в свою пользу одну из компонент дополнительного дохода, тогда как вторая компонента распределяется между франчайзи и франчайзером пропорционально их вкладу. Иными словами:

$$
B=1, W_{\text {sup }}>W_{\text {ind }}, \quad C=\frac{P_{\text {sup }}}{P_{\text {sup }}+P_{\text {ind }}},
$$

или:

$$
C=1, \quad P_{\text {sup }}>P_{\text {ind }}, \quad B=\frac{W_{\text {sup }}}{W_{\text {sup }}+W_{\text {ind }}} .
$$

3. Один из множителей принимается равным нулю, а второй - равным единице. Это означает, что один из компонентов дополнительного дохода полностью отходит к франчайзеру, а второй - к франчайзи. Этот подход прост и удобен для практических целей, однако пользоваться им надо с определенной осторожностью. Дело в том, что если ситуация, при которой

$$
\begin{aligned}
& B=1, \quad W_{\text {sup }}>>W_{\text {ind }}, \\
& C=0, \quad P_{\text {ind }}>>P_{\text {sup }}
\end{aligned}
$$


приемлема для франчайзи, так как дает ему защиту от рисков, то ситуация, когда

$$
\begin{aligned}
& C=1, \quad \begin{array}{l}
P_{\text {sup }}>P_{\text {ind }}, \\
B=0,
\end{array}, W_{\text {ind }}>>W_{\text {sup }}
\end{aligned}
$$

для франчайзи в общем случаем неприемлема и служит указанием на то, что вхождения в сеть данного франчайзера следует избегать, так как он не дает достаточных гарантий успеха. Иными словами, при использовании этого алгоритма определения долевых множителей допустимо только $B=1, C=0$.

4. $\quad$ Оба долевых множителя $B$ и $C$ равны 0. Этот метод может быть реализован в двух вариантах:

- В первом случае:

$$
C=0, P_{\text {ind }}>>P_{\text {sup }} .
$$

$$
B=0, W_{\text {ind }}>>W_{\text {sup }},
$$

Очевидно, что этот вариант не имеет никакого экономического смысла, так как приобретение франшизы не дает франчайзи никаких преимуществ по сравнению с ведением независимой предпринимательской деятельности. В этой ситуации ставка роялти (если предположить, что найдется франчайзи, желающий купить такую франшизу), рассчитанная по формуле (7), также будет равна нулю (или, точнее, с математической точки зрения она будет пренебрежимо мала, потому что, хотя $W_{\text {sup }} P_{\text {sup }}<<W_{f r} P_{f r}$, тем не менее, $W_{\text {sup }} P_{\text {sup }} \neq W_{f r} P_{f r}$; однако с экономической точки зрения ее следует принять равной нулю, потому что выгоды франчайзи не получает).

- Второй вариант установления таких значений долевых множителей имеет не расчетно-экономическую, а управленческую природу. Иными словами, франчайзер, хотя условие (9) и не выполняется, добровольно ограничивает свой доход, стимулируя тем самым своих франчайзи. Этот вариант удобнее всего, по нашему мнению, реализовывать в виде фиксированных отчислений (см. формулу (6)).

5. $\quad$ Множители $B$ и $C$ рассчитываются по формуле

$$
B=C=\max \left\{\frac{W_{\text {sup }}}{W_{\text {sup }}+W_{\text {ind }}} ; \quad \frac{P_{\text {sup }}}{P_{\text {sup }}+P_{\text {ind }}}\right\} .
$$

Эта схема соответствует желанию франчайзера увеличить свой доход за счет франчайзи. составит:

Дополнительный доход франчайзера $R_{a d d}$ в этом случае по сравнению с формулой (7)

$$
\begin{aligned}
& R_{a d d}=\left[\max \left\{\frac{W_{\text {sup }}}{W_{\text {sup }}+W_{\text {ind }}} ; \frac{P_{\text {sup }}}{P_{\text {sup }}+P_{\text {ind }}}\right\}-\frac{W_{\text {sup }}}{W_{\text {sup }}+W_{\text {ind }}}\right] W_{\text {sup }} P_{\text {ind }}+ \\
& +\left[\max \left\{\frac{W_{\text {sup }}}{W_{\text {sup }}+W_{\text {ind }}} ; \frac{P_{\text {sup }}}{P_{\text {sup }}+P_{\text {ind }}}\right\}-\frac{P_{\text {sup }}}{P_{\text {sup }}+P_{\text {ind }}}\right] W_{\text {ind }} P_{\text {sup }}
\end{aligned}
$$

Очевидно, что как минимум одно из слагаемых в этой формуле будет равно 0.

Вероятно, такая схема может быть рекомендована для использования на стабильных рынках, где торговая марка франчайзера хорошо известна и служит гарантией привлечения покупателей.

6. Множители $B$ и $C$ рассчитываются по формуле: 
(11)

$$
B=C=\min \left\{\frac{W_{\text {sup }}}{W_{\text {sup }}+W_{\text {ind }}} ; \frac{P_{\text {sup }}}{P_{\text {sup }}+P_{\text {ind }}}\right\} .
$$

Эта модель, напротив, позволяет франчайзи увеличить свой доход. Она может применяться на сложных рынках, ведение бизнеса на которых требует больших усилий от франчайзи (например, при запуске франчайзинговой сети на рынке иностранного государства, где бренд франчайзера неизвестен; в этой ситуации франчайзи сложнее реализовать весь потенциал франшизы, чем на тех рынках, где соответствующий бренд хорошо известен).

Доход $R_{l}$, который франчайзер недополучит в этом случае по сравнению с базовой методикой, может быть найден по формуле:

$$
\begin{aligned}
& R_{l}=\left[\frac{W_{\text {sup }}}{W_{\text {sup }}+W_{\text {ind }}}-\min \left\{\frac{W_{\text {sup }}}{W_{\text {sup }}+W_{\text {ind }}} ; \frac{P_{\text {sup }}}{P_{\text {sup }}+P_{\text {ind }}}\right\}\right] W_{\text {sup }} P_{\text {ind }}+ \\
& +\left[\frac{P_{\text {sup }}}{P_{\text {sup }}+P_{\text {ind }}}-\min \left\{\frac{W_{\text {sup }}}{W_{\text {sup }}+W_{\text {ind }}} ; \frac{P_{\text {sup }}}{P_{\text {sup }}+P_{\text {ind }}}\right\}\right] W_{\text {ind }} P_{\text {sup }}
\end{aligned}
$$

Отметим, что перечисленные варианты задания значений множителей $B$ и $C$ представляют собой наиболее простые с математической точки зрения и наиболее очевидные и прозрачные с экономической точки зрения способы. На практике, разумеется, франчайзер может задать их значение иным способом, отталкиваясь от своих целей и задач. Отметим, что значения $B$ и $C$ задаются именно франчайзером, так как условия предоставления франшизы определяет он.

Не следует думать, что описанная методика расчета ставки роялти имеет исключительно теоретический характер - на рынках с давними традициями франчайзинга существует достаточно подробная статистика, позволяющая определить, каков дополнительный доход франчайзи от использования переданной ему ИС, насколько выше вероятность выживания франчайзи по сравнению с независимым предпринимателем и каков средний доход независимого предпринимателя. В ряде случаев соответствующую статистику собирают сами франчайзеры. И поэтому (особенно это справедливо для крупных сетей, заинтересованных в эффективном управлении доходами от франчайзи и обладающих необходимыми организационными и информационными ресурсами для создания и функционирования такой системы) подготовка базовой информации для расчета по приведенной выше методике значительных затруднений не представляет.

Если франчайзер желает взимать не только роялти, но и первоначальный взнос, то в этом случае ставка роялти может быть найдена путем решения следующего уравнения:

$$
\frac{r}{100 \%} R_{t o t}=I F+\frac{r_{I F}}{100 \%} R_{t o t}
$$

где

$r$ - ставка роялти (рассчитанная по формуле (7));

$R_{\text {tot }}$ - суммарный доход, полученный франчайзи в течение срока действия франчайзингового договора;

$I F$ - первоначальный взнос (его величина устанавливается франчайзером);

$r_{I F}$ - ставка роялти (с учетом взимания первоначального взноса)).

\section{Выводы}

Наиболее важным недостатком существующих моделей расчета величины платы за использование ИС является, на наш взгляд, постулат об их универсальном характере предполагается, что одна и та же модель может быть применена для любых форм торговли 
ИС. Нашей целью было показать, что это не так, и при расчете ставки роялти необходимо принимать во внимание природу тех преимуществ, которые получает пользователь ИС. Это особенно важно при определении механизма финансовых отношений между франчайзи и франчайзером, так как преимущества франчайзи от приобретения франшизы не сводятся только к возможности получить дополнительный доход - он также снижает риски своей деятельности благодаря использованию торговой марки франчайзера и получению доступа к его консультационной поддержке.

Изложенная в данной статье модель вводит в расчет величины роялти при франчайзинге плату за снижение риска. Безусловно, речь идет о базовой модели, нуждающейся в доработке (например, желательно учесть в ней риски франчайзера, связанные с недобросовестным поведением франчайзи). Тем не менее автор надеется, что она сможет послужить как удобным инструментом для практиков, работающих в сфере франчайзинга, так и хорошей основой для построения более сложных моделей для теоретиков.

\section{Список литературы}

1. Алгазина Д.Г. Особенности определения стратегии ценообразования в производственно-торговых франчайзинговых системах // Известия Алтайского Государственного Университета. 2005. № 2 (36). С. 12-14.

2. Королева Н.С. Показатели и критерии оценки эффективности франчайзинга в предпринимательской деятельности // Вестник ИНЖЭКОНа. 2006. № 4 (13). С. 267268.

3. Котляров И.Д. Математическая модель принятия решения о приобретении франшизы // Проблемы управления. 2007. № 5. С. 84-87.

4. Стажкова М.M. (2007). Договор франчайзинга: правовые основы, учеты и налоги. М.: ООО ИИА «Налог Инфо», ООО «Статус Кво 97».

5. Blair, R.D., Lafontaine, F. (2005), The Economics of Franchising. New York. 